University of New Hampshire

University of New Hampshire Scholars' Repository

Space Science Center

Institute for the Study of Earth, Oceans, and

Space (EOS)

2006

\title{
Optimization of Single-Sided Charge-Sharing Strip Detectors
}

\author{
L A. Hamel \\ University of Montreal \\ M Benoit \\ University of Montreal \\ B Donmez \\ University of New Hampshire - Main Campus \\ John R. Macri \\ University of New Hampshire - Main Campus, John.Macri@unh.edu \\ Mark L. McConnell \\ University of New Hampshire - Main Campus, mark.mcconnell@unh.edu
}

See next page for additional authors

Follow this and additional works at: https://scholars.unh.edu/ssc

Part of the Astrophysics and Astronomy Commons

\section{Recommended Citation}

Hamel, L.A.; Benoit, M.; Donmez, B.; Macri, J.R.; McConnell, M.L.; Narita, T.; Ryan, J.M., "Optimization of Single-Sided Charge-Sharing Strip Detectors," Nuclear Science Symposium Conference Record, 2006. IEEE , vol.6, no., pp.3759,3761, Oct. 29 2006-Nov. 12006

This Conference Proceeding is brought to you for free and open access by the Institute for the Study of Earth, Oceans, and Space (EOS) at University of New Hampshire Scholars' Repository. It has been accepted for inclusion in Space Science Center by an authorized administrator of University of New Hampshire Scholars' Repository. For more information, please contact Scholarly.Communication@unh.edu. 


\section{Authors}

L A. Hamel, M Benoit, B Donmez, John R. Macri, Mark L. McConnell, T Narita, and James M. Ryan 


\title{
Optimization of Single-Sided Charge-Sharing Strip Detectors
}

\author{
L.A. Hamel, M. Benoit, B. Dönmez, J. R. Macri, M. L. McConnell, T. Narita and J. M Ryan
}

\begin{abstract}
Simulation of the charge sharing properties of single-sided CZT strip detectors with small anode pads are presented. The effect of initial event size, carrier repulsion, diffusion, drift, trapping and detrapping are considered. These simulations indicate that such a detector with a $150 \mu \mathrm{m}$ pitch will provide good charge sharing between neighboring pads. This is supported by a comparison of simulations and measurements for a similar detector with a coarser pitch of $225 \mu \mathrm{m}$ that could not provide sufficient sharing. The performance of such a detector used as a gamma-ray imager is discussed.
\end{abstract}

\section{INTRODUCTION}

$\mathrm{S}_{\mathrm{i}}$ charge-sharing strip detectors have recently been proposed as a way to use coplanar strips with collecting electrodes only [1-4]. Such detectors have some of the advantages of pixel detectors for gamma-ray imaging but need far fewer electronics channels. This feature is particularly interesting for space applications. The structure of the anode is identical to a pixel detector i.e. it is made of closely packed square pad contacts. These contacts are then interconnected in columns and rows in the layers of the carrier substrate in order to form two sets of orthogonal readout channels.

All these electrode pads are biased at the same potential and are all collecting anodes. The principle of operation requires sharing of the electron charge between row and column electrodes for each event. This is feasible when the lateral extent of the electron cloud exceeds the pitch of the anode contact pads (see Fig. 1).

Results obtained with a first prototype with a $225 \mu \mathrm{m}$ pitch have been reported earlier [1-4]. It was observed that, for a large fraction of the events, most of the charge was collected on only one readout channel, indicating that the pitch was still too coarse.

This paper presents simulations of the various processes involved in the electron collection to evaluate the size of the electron distribution when the electrons are collected at the anode surface. Results of such simulations are presented for the $225 \mu \mathrm{m}$ pitch prototype that reproduce the measurements. Additional simulation results are shown for a similar detector with a pitch of $150 \mu \mathrm{m}$ that indicate that such a detector should provide improved imaging efficiency.

Manuscript recved November 17, 2006

L.A. Hamel and M. Benoit are with the Université de Montréal, Montréal, Canada, (phone: 514-343-6204, e-mail: louis-andre.hamel @umontreal.ca).

B. Dönmez, J. R. Macri, M. L. McConnell and J. M Ryan are with the Space Science Center, University of New Hampshire, Durham, NH 03824. T. Narita is with the College of the Holy Cross, Worcester, MA 01610.

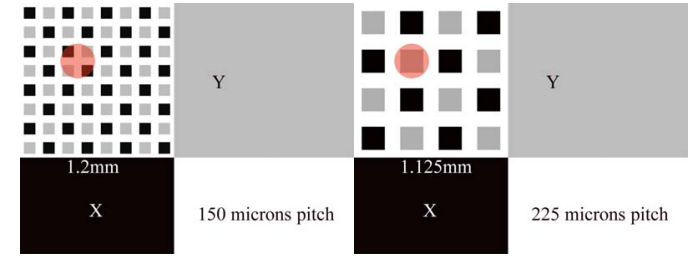

Fig. 1. Charge sharing between pixels is determined by the pixel pitch and by the electron cloud size (red circle).

\section{Simulation AlgORITHM}

A simulation package has been developed that tracks the evolution of the electron cloud from its generation by a gamma-ray interaction to its collection at the anode surface. The effects of extraction from the electron-hole cluster, drift in the electric field, trapping, trapping-detrapping on shallow states, diffusion and electrostatic repulsion between the electrons in the cloud have been considered.

\section{A. Gamma-ray interaction}

The physics of the radiation interaction with the detector can be simulated by GEANT4, but for this work we are only interested in the evolution of the electron cloud size after the interaction. It was therefore sufficient to simulate only the depth of the first interaction in CZT. Electron-hole clusters were thus randomly generated according to an exponentially decreasing distribution in the $z$-direction, using the total interaction cross-section at a given energy. The clusters were generated uniformly across the unit cell in the other directions. Secondary interactions were not considered. The clusters were taken as spherically symmetric Gaussian distributions of electron-hole pairs with an initial rms radius of $5 \mu \mathrm{m}$ in every direction. A more detailed simulation is easily implemented when needed.

\section{B. Electron-hole separation}

The time needed to extract the electrons from the clusters was found to be of the order of several nanoseconds and was therefore neglected. Holes were considered fixed.

\section{Electron drift, trapping and detrapping}

The electric field map in our detector is calculated using COMSOL Multiphysics [5]. The electrons are transported along the electric field lines by a numerical integration over small time steps. Deep trapping with a lifetime of $4 \mu \mathrm{s}$ and trapping and detrapping on a shallow state, with trapping and detrapping times of $40 \mathrm{~ns}$ and $10 \mathrm{~ns}$ respectively, are simulated by Monte Carlo during each integration step. The trappingdetrapping process provides dispersion in the transport. 


\section{Repulsion and diffusion}

After each time step, each electron is displaced randomly to simulate the effects of diffusion and electrostatic repulsion. While diffusion is a stochastic process, repulsion is not and must involve a sum over every charge pair in the cloud. But it is easily found that, for a cloud with ellipsoidal symmetry, only the dimensions of the ellipsoid is affected by the repulsion. In this approximation, the cumulative effects of diffusion and repulsion can be combined by introducing an effective diffusion coefficient

$$
D_{\text {eff }}=D+\frac{1}{15}\left(\frac{3 \mu N e}{4 \pi \varepsilon}\right) \frac{1}{\sqrt{5} \sigma}
$$

where $D$ is the diffusion coefficient, $\mu$ is the mobility, $N$ is the number of electrons in the cloud, $e$ is the electron charge, $\varepsilon$ is the CZT permittivity and $\sigma$ is the instantaneous rms radius of the cloud in $x, y$ or $z$. $D_{\text {eff }}$ is an implicit function of time and decreases when the cloud expands. This approximation was tested by comparing its results with full calculations involving the actual electric force on every electron pair in the cloud. Even for cloud shapes that are not ellipsoidal, the difference is barely discernable. Fig. 2 shows the effects of diffusion, repulsion and their combined effect for an electron cloud produced by $1 \mathrm{MeV}$ point-like energy deposit in CZT. The combined effect is approximately given by the quadratic sum of each taken separately.

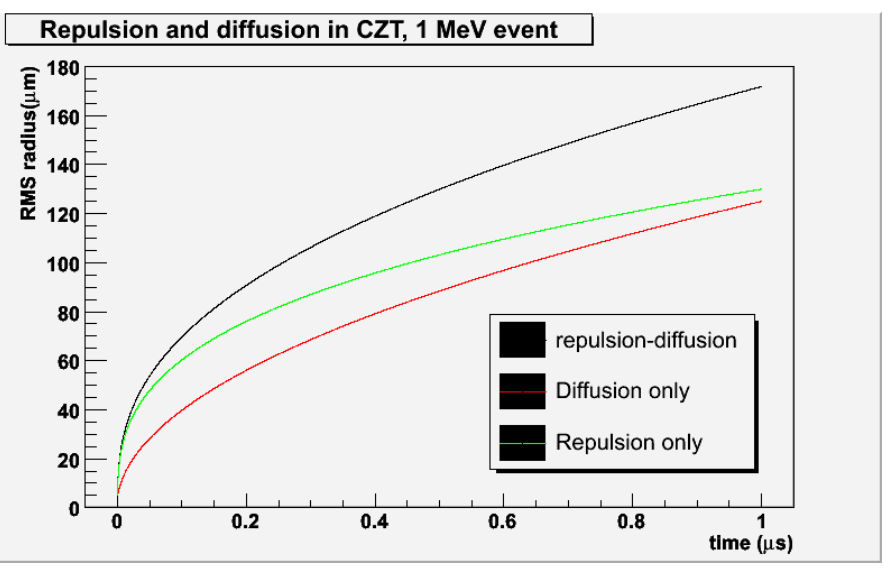

Fig. 2. Approximated repulsion and diffusion curve, for a $1 \mathrm{MeV}$ event

\section{E. Signals}

After each time step, the induced charge signals in the various electrodes are calculated by summing the contribution of each electron in the cloud. This involves the weighting potentials for each readout electrode (Fig. 3). These potentials are again calculated by COMSOL Multiphysics. The charge induced at the end of the simulation, when all electrons are either collected at the anode or deeply trapped, is the net charge measured by that electrode.

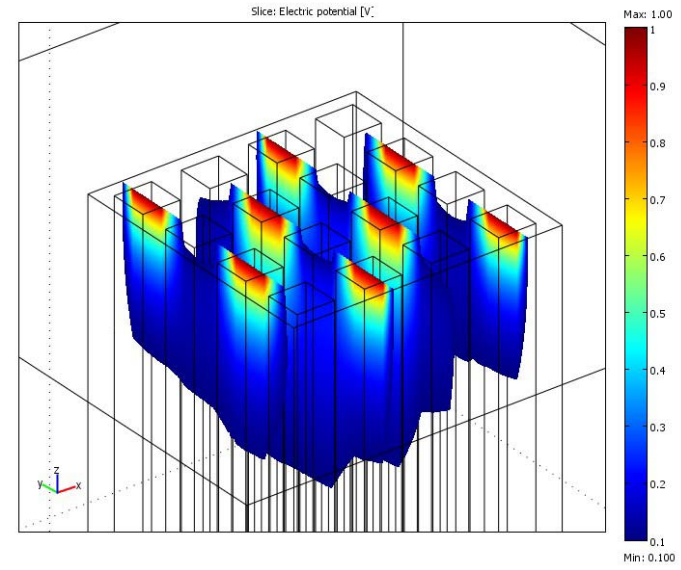

Fig. 3. Weighting potential for one (X or Y) readout electrode.

\section{RESULTS OF THE SIMULATION}

Fig. 4 shows experimental results for our present $225 \mu \mathrm{m}$ pitch prototype illuminated from the cathode side by a ${ }^{57} \mathrm{Co}$ source through a $1 \mathrm{~mm}$ diameter collimator centered on a unit cell. The scatter plot shows the charge sharing fraction between the $\mathrm{X}$ and $\mathrm{Y}$ readout channels. The energy threshold on each channel is $15 \mathrm{keV}$. Photopeak events were selected. The large number of events recorded with most of the charge collected on either the $\mathrm{X}$ or $\mathrm{Y}$ channel indicates that the 225 $\mu \mathrm{m}$ pitch is too coarse.

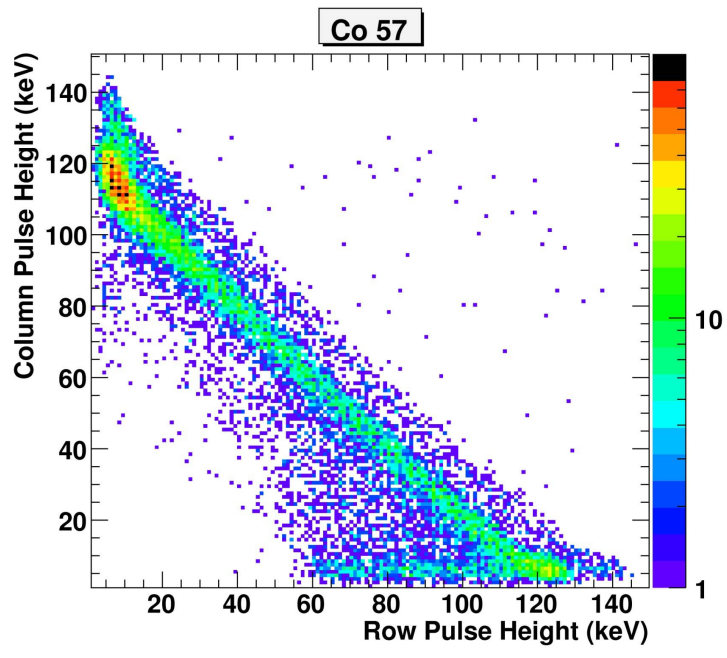

Fig. 4. Experimental scatter plot for a 225 micron detector for a ${ }^{57} \mathrm{Co}$ spectrum.

Fig. 5 shows the simulated results for the same situation. Although the simulated scatter plot has poorer statistics and contains no Compton scattering (only the $122 \mathrm{keV}$ line), it agrees with the data. Here again, most events share little significant charge with neighboring electrodes. Other comparisons at different energies exhibit the same feature. Despite some approximations, the simulation successfully reproduces the experimental results for the $225 \mu \mathrm{m}$ pitch prototype. 


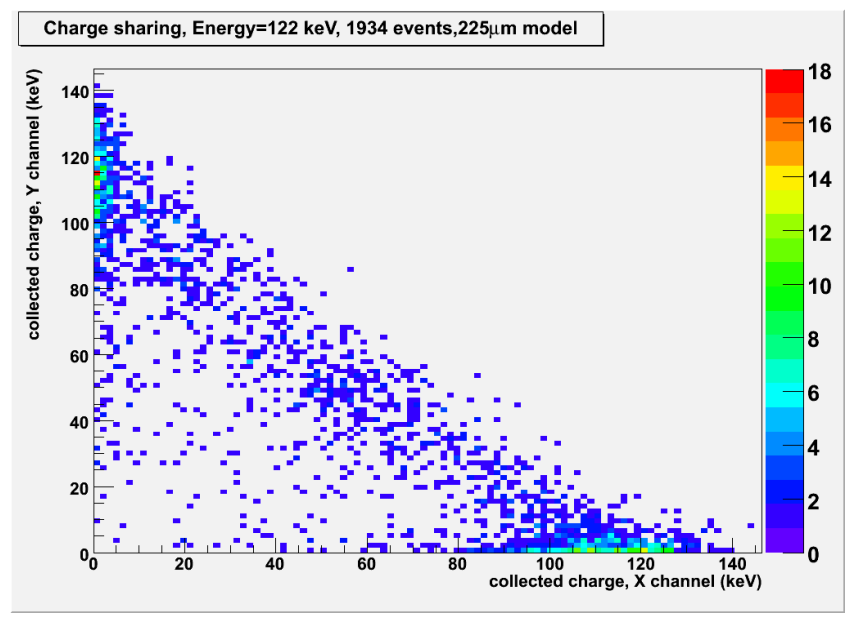

Fig. 5. Simulated scatter plot for a 225 micrometer detector for a Co 57 spectrum $(122 \mathrm{keV})$

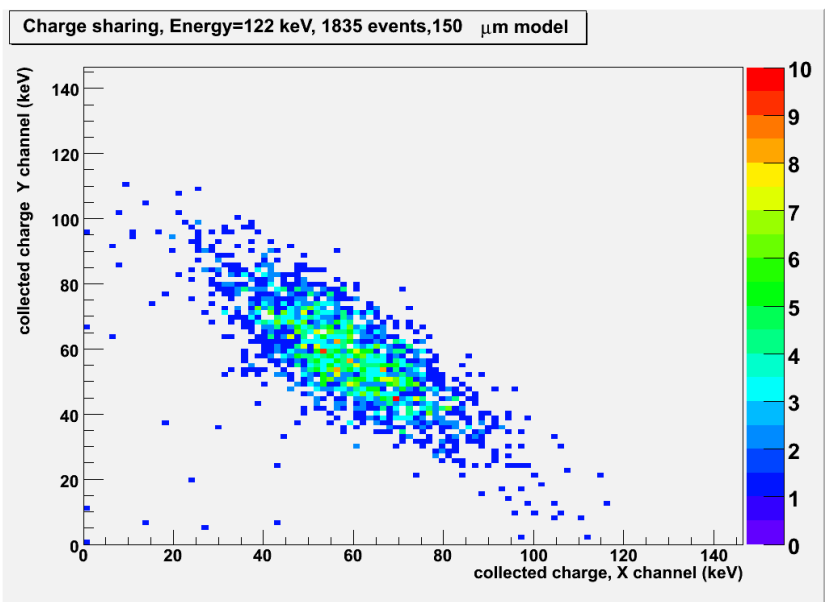

Fig. 6. Simulated scatter plot for a 150 micron detector at $122 \mathrm{keV}$.

Fig. 6 shows results of the same simulation but for a hypothetical detector with a reduced pitch of $150 \mu \mathrm{m}$. This time, the scatter plot reveals a different behavior. The distribution now clusters at the midpoint, indicating improved charge sharing between neighboring electrodes. Since the imaging efficiency is directly related to the probability that an event trigger both the $\mathrm{X}$ and the $\mathrm{Y}$ readout channels, this result predicts a significant improvement of the detector efficiency at a reduced pitch of $150 \mu \mathrm{m}$.

\section{CONCLUSION}

To study and understand the charge sharing behavior of our coplanar strip detectors, we developed a simulation package to investigate the time evolution of the electron cloud during the collection process. The simulation faithfully reproduces the experimental results for a $225 \mu \mathrm{m}$ pitch detector.

The simulation tool was then used to predict the charge collection behavior in a similar detector with a reduced pitch of $150 \mu \mathrm{m}$. These calculations indicate a level of charge sharing that fulfills the intent of the electronic design, i.e., that charge is comparably shared between neighboring electrodes, thus providing much improved event location and imaging efficiency.

Efforts are underway in the fabrication of such a detector.

\section{REFERENCES}

[1] J.R. Macri, L.A. Hamel, M. Julien, B. Dönmez, M.L. McConnell, J.M. Ryan, M. Widholm, and T. Narita, "Single-sided charge-sharing CZT strip detectors", IEEE Rome.

[2] B. Dönmez, J.R. Macri, M.L. McConnell, J.M. Ryan, M. Widholm, T. Narita and L.A. Hamel, "Further studies of single-sided chargesharing CZT strip detectors", SPIE 2005.

[3] B. Dönmez, J.R. Macri, M.L. McConnell, J.M. Ryan, M. Widholm, L.A. Hamel and T. Narita, "Continued studies of single-sided charge-sharing CZT strip detectors", IEEE 2005.

[4] B. Dönmez, J.R. Macri, J.M. Ryan, J.S. Legere, M.L. McConnell, M. Widholm, T. Narita and L.A. Hamel, "Characterization of singlesided charge-sharing CZT strip detectors for gamma-ray astronomy", Proc. SPIE, 2006

[5] COMSOL Multiphysics 3.3, COMSOL AB. 